\title{
Pegase: a space-based nulling interferometer
}

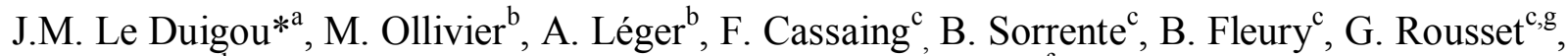

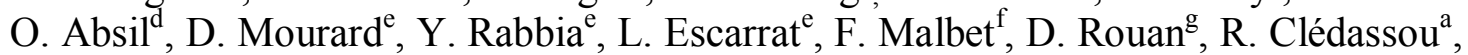 \\ M. Delpech ${ }^{\mathrm{a}}$, P. Duchon ${ }^{\mathrm{a}}$, B. Meyssignac ${ }^{\mathrm{a}}$, P.-Y. Guidotti ${ }^{\mathrm{a}}$, N. Gorius ${ }^{\mathrm{a}}$

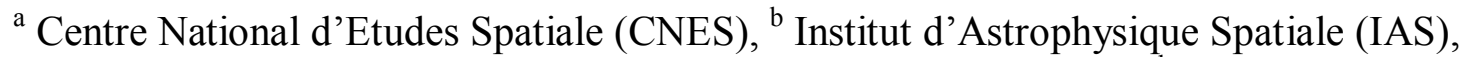 \\ ${ }^{c}$ Office National d'Etudes et de Recherches Aéronautiques (ONERA), ${ }^{d}$ Université de Liège, \\ ${ }^{\mathrm{e}}$ Observatoire de la Côte d'Azur (OCA), ${ }^{\mathrm{f}}$ Laboratoire d'Astrophysique de l'Observatoire de \\ Grenoble (LAOG), ${ }^{\mathrm{g}}$ Observatoire de Paris
}

\begin{abstract}
The space based mission Pegase was proposed to CNES in the framework of its call for scientific proposals for formation flying missions. This paper presents a summary of the phase- 0 performed in 2005 . The main scientific goal is the spectroscopy of hot Jupiters (Pegasides) and brown dwarfs from 2.5 to $5 \mu \mathrm{m}$. The mission can extend to other objectives such as the exploration of the inner part of protoplanetary disks, the study of dust clouds around AGN,... The instrument is basically a two-aperture $(\mathrm{D}=40 \mathrm{~cm})$ interferometer composed of three satellites, two siderostats and one beamcombiner. The formation is linear and orbits around L2, pointing in the anti-solar direction within a $+/-30^{\circ}$ cone. The baseline is adjustable from 50 to $500 \mathrm{~m}$ in both nulling and visibility measurement modes. The angular resolution ranges from 1 to 20 mas and the spectral resolution is 60 . In the nulling mode, a $2.5 \mathrm{~nm}$ rms stability of the optical path difference (OPD) and a pointing stability of 30 mas rms impose a two level control architecture. It combines control loops implemented at satellite level and control loops operating inside the payload using fine mechanisms. According to our preliminary study, this mission is feasible within an 8 to 9 years development plan using existing or slightly improved space components, but its cost requires international cooperation. Pegase could be a valuable Darwin/TPF-I pathfinder, with a less demanding, but still ambitious, technological challenge and a high associated scientific return.
\end{abstract}

Keywords: Interferometry, Nulling, Formation flying, Cophasing, Spectroscopy, low mass objects, Protoplanetary disks.

\section{1 - INTRODUCTION}

In 2004, CNES issued a call for scientific proposals for space based formation flying missions. A consortium led by IAS and including other scientific laboratories and Alcatel Alenia Space, proposed the Pegase mission (Leyre, 2004 ${ }^{1}$. It was selected to proceed to a phase 0 process in 2005 . This paper summarizes the work performed in this framework by a joint team of CNES and ONERA engineers and scientists from various laboratories.

\section{2 - SCIENTIFIC OBJECTIVES}

\section{1 - Hot Jupiters}

During the past decade, the indirect ground based observations proved the existence of more than 150 giant exo-planets. A special class is composed by hot Jupiters (HJ) which orbit very close around their parent star (from 0.05 to $0.1 \mathrm{AU}$ ). Their mass varies from 0.11 to 10 mass of Jupiter $\left(\mathrm{M}_{\mathrm{J}}\right)$ and their temperature reaches 700 to $1500 \mathrm{~K}$. Their formation process remains poorly understood and there is now room for a new science aiming at a better understanding of such objects. The Corot and Kepler missions, thanks to the transit detection method combined with available ground information, will provide us with precise estimates of the mass and the radius of such objects. The Pegase mission is interested by the next step which is the spectral characterization of hot Jupiters (also called Pegasides, which give the name of the mission). As shown by figure 1, the current models need to be constrained by measurements to better characterize the physics of their atmosphere: the chemical composition, the amount of thermalization, the existence of local winds, the importance of clouds, ...The main molecular signatures of interest $\left(\mathrm{CH}_{4}, \mathrm{H}_{2} \mathrm{O}, \mathrm{CO}\right)$ lie in the $1.5-5 \mu \mathrm{m}$ range. The required spectral resolution is moderate $(\mathrm{R}=60)$ and the minimum SNR is 10 .

* Jean-Michel.LeDuigou@enes.fr tel : (+33) 561282124 fax : (+33) 56128 26 92, 18 Av Edouard Belin, 31401 Toulouse, France 

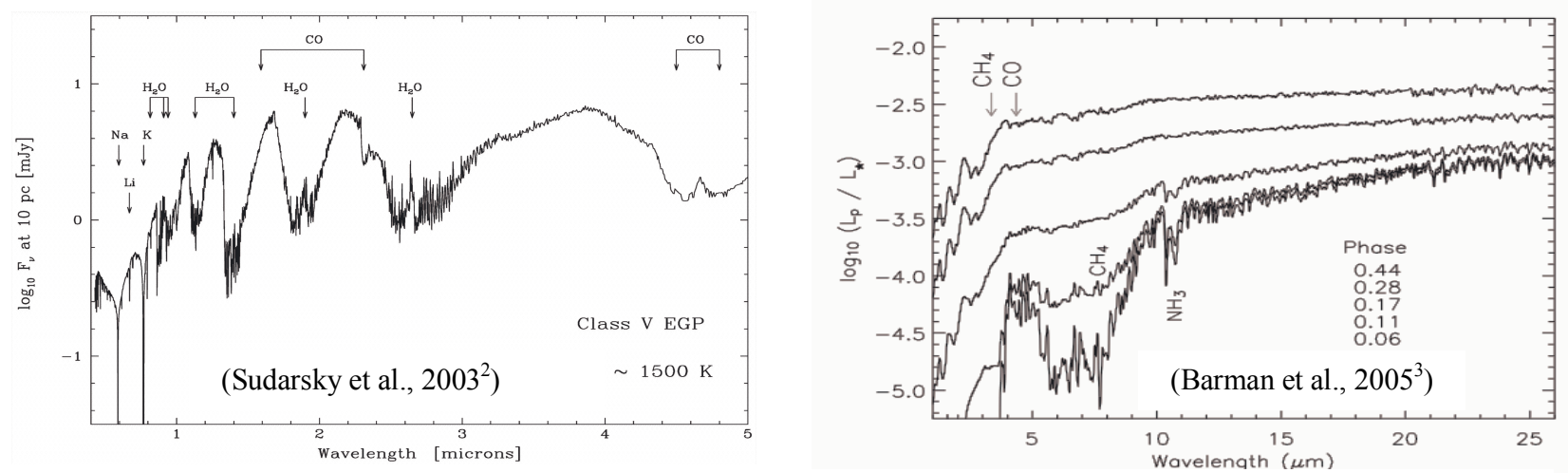

Figure 1: atmospheric models of hot Jupiters. Left: low thermalization. Right: high thermalization

The main instrumental challenge is to achieve a very high angular resolution ( 0.5 to 5 mas) together with a high extinction power of the parent star light. The contrast (star/planet flux ratio) ranges from $10^{3}$ to a few $10^{4}$ in the considered wavelength range. As the desired angular resolution at 2.5-5 $\mu \mathrm{m}$ implies aperture dimensions of several hundred meters and the terrestrial atmospheric absorption limits the ground based observations to some narrow bands, a space based free flying interferometer is proposed. The desired extinction can be implemented using the Bracewell set up (Bracewell, $1978^{4}$ ), i.e. a Michelson interferometer with a $\pi$ achromatic phase shift in one arm and a rotation of the baseline around the line of sight. Figure 2 shows how this concept projects a dark fringe on the central parent star while the baseline is adjusted so that a bright fringe coincides with the supposed weak companion elongation. As shown by many authors (Léger, $1997^{5}$, Angel, $1997^{6}$ ), this concept can not be directly applied to the telluric exo-planets spectral characterization in the mid IR because the nulling ratio of a two apertures system is insufficient to face the $10^{6}$ contrast at $10 \mu \mathrm{m}$ and a centro-symetric sub-aperture pattern can not extract the exo-planet signal from the exo-zodiacal light. These restrictions no more apply in the case of Pegase because the planet flux is much stronger and the exo-zodiacal light weaker in the selected wavelength range. From the initial list, 16 hot Jupiters were identified as good candidates for the mission. This number may increase in the next years, but will reach a maximum when all nearby stars will have been scanned by indirect methods.

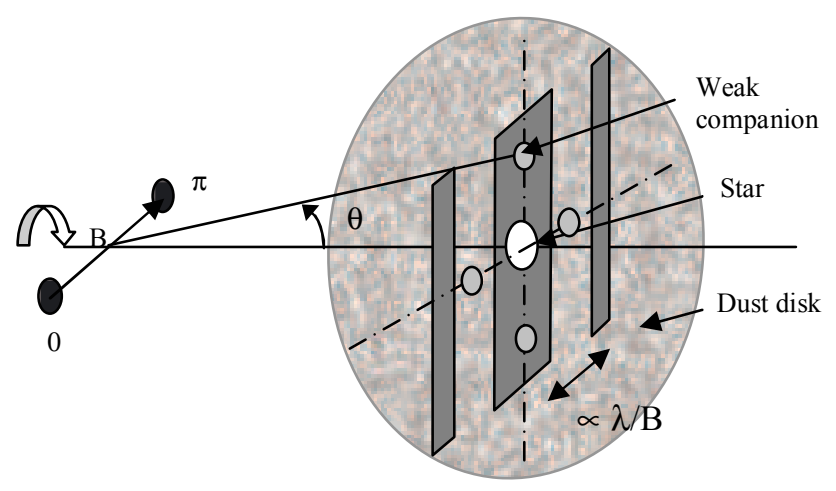

Figure 2: basic principle of a Bracewell interferometer.

\section{2 - Brown dwarfs}

Reducing the size of the baseline to adapt to greater elongations (10 to 100 mas), the same instrument can also characterize the atmosphere of brown dwarfs (BD) using nulling interferometry. This is the second main objective of Pegase which consists mainly in exploring the brown dwarfs desert and constraining the current models of these objects: the relationship between the effective temperature and the radius as a function of mass, the importance of the atmosphere, and especially of $\mathrm{CH}_{4}$ and clouds. The brown dwarfs have the same kind of characteristic spectral signatures as compared to the hot Jupiters, but their mass lies in the 10 to $80 \mathrm{M}_{\mathrm{J}}$ range. The contrast with the parent star depends on the mass of the brown dwarf and of the age of the system. For masses between 15 and $80 \mathrm{M}_{\mathrm{J}}$ and systems younger than 1 billion years, the contrast ranges from $10^{3}$ to $10^{4}$. Furthermore, the measurements have to be combined with a precise mass estimate to test the coherence of the atmosphere and internal structure models. These requirements limit currently the number of possible targets to a few ones. But future on ground observations will probably extend the list.

\section{3 - Other objectives}

If the nulling mode is complemented by a more classical but very precise interferometry mode measuring the visibility of the fringes $\left(\mathrm{V}^{2}\right.$ mode), one can adapt the Pegase instrument to various other valuable scientific objectives such as the study of circumstellar disks, the study of dust distribution around AGN, the measure of the distance of the Magellan cepheids, the study of massive hydrogen clouds in front of quasars,... To study the $\mathrm{V}^{2}$ mode in the phase 0 , we selected 
one of the most interesting features: the possible detection of gaps in protoplanetary disks around T-Tauri and Herbig He-Be stars located in star formation regions like the Taurus one $(150 \mathrm{pc})$. Most of the recent studies tend to indicate that the planets formation process begins well before the parent star reaches the main sequence, with probable migrations from the external to the internal regions. During this process each planet should create a characteristic signature, such as a gap, in the dust cloud that slightly modifies the IR emission. Hence, the detailed study of circumstellar disks around young objects is a good test for the presence of planets if one can precisely detect such gaps. Preliminary simulations show that a 0.05 AU gap induces variations of the visibility of a few \% (see figure 4 ).

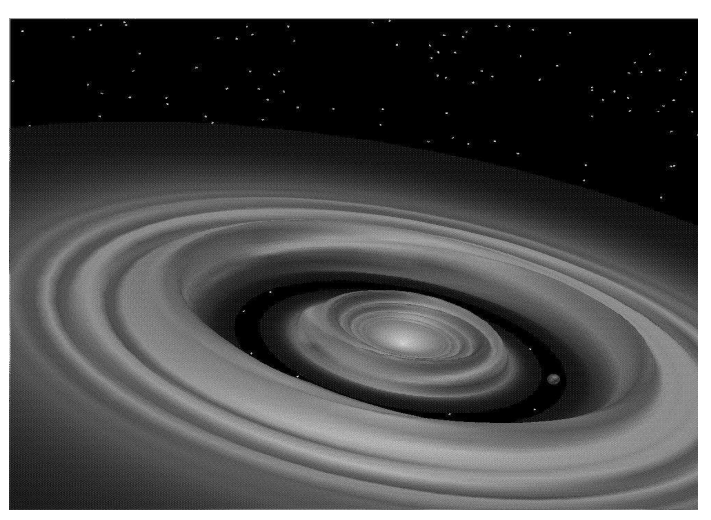

Figure 3: artist's view of gap in a circumstellar disk

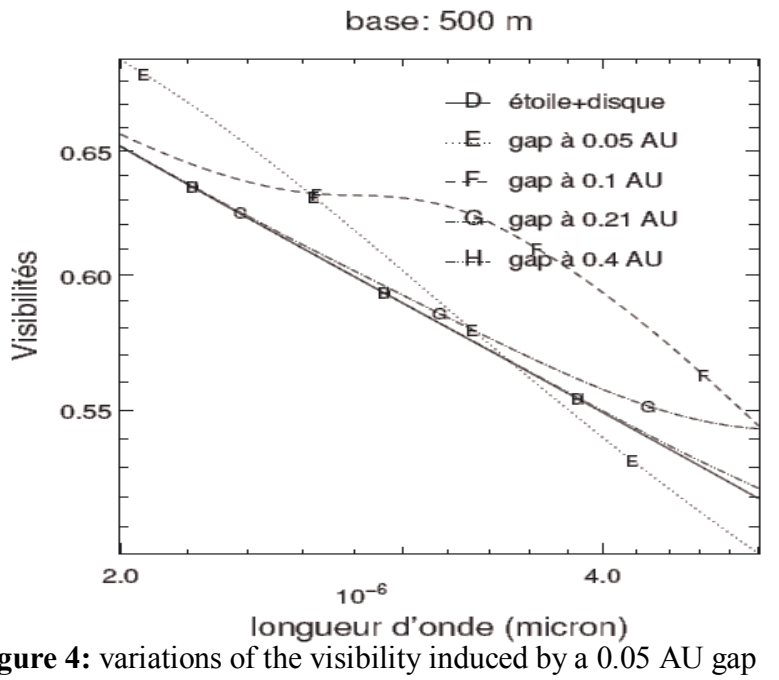

Figure 4: variations of the visibility induced by a 0.05 AU
around a classical T-Tauri star (CTSS model) $B=500 \mathrm{~m}$

\section{3 - SNR CALCULATIONS AND MISSION MAIN PARAMETERS}

Simplified SNR calculations were performed in the scientific laboratories and at CNES with very consistent results. As an example, we detail here below the case of the observation of the hot Jupiters in the nulling mode. Some numerical results are provided in a reference case which is the observation at $3 \mu \mathrm{m}$ of Ups And b with the parameters of figure 5.

We suppose that the size and orientation of the baseline are adjusted to the previously known planet elongation so that we have a maximum transmission at $2.5 \mu \mathrm{m}$ on the planet. Another major assumption is the use of single mode fibers to clean the wavefronts and relax the constraints on optical surfaces (Ollivier, 1997 ${ }^{7}$ ). This process converts differential wavefront errors into intensity mismatch (Ruillier, $2001^{8}$ ) which have less impact on the nulling performance than phase errors. After spatial filtering, the residual star light, the planet light and the environmental noises are focused on one pixel for each spectral channel. The average values of noises are removed by a calibration process which includes measurements with at least two angular positions around the line of sight.

The emission of the planet in one spectral channel of width $\Delta \lambda$ is modelled by the following equation:

$$
I_{p} \approx \pi \eta_{o}(\lambda) \eta_{q}(\lambda) \eta_{p}(\lambda, B) A \Delta \lambda\left[\frac{A_{p} R_{p}^{2}}{4 a_{p}{ }^{2}}\left(\frac{R_{*}}{d}\right)^{2} B_{\lambda}\left(T_{*}\right)+\left(\frac{R_{p}}{d}\right)^{2} S_{p}(\lambda) B_{\lambda}\left(T_{p}\right)\right]
$$

$\eta_{\mathrm{p}}(\lambda, \mathrm{B})$ is the interferometric transmission at the elongation of the planet: $\eta_{\mathrm{p}}(\lambda, \mathrm{B})=2 \sin ^{2}(\mathrm{X})$ with $\mathrm{X}=\pi \mathrm{B} \Delta \theta * / \lambda$. $\mathrm{T}_{\mathrm{p}}$ and $\mathrm{T} *$ are respectively the planet and star effective temperature, $R_{p}$ and $R_{*}$ the associated radius, $S_{p}$ the absorption spectrum, $A_{p}$ the albedo coefficient, $a_{p}$ the planet elongation, $\eta_{\mathrm{o}}(\lambda)$ the optical transmission for one arm, $\eta_{\mathrm{q}}(\lambda)$ the quantum efficiency of the detector, A the collecting area, $\mathrm{B}$ the baseline. $\mathrm{B}_{\lambda}(\mathrm{T})$ is the Planck function. In the reference case $<\mathrm{I}_{\mathrm{p}}>=23 \mathrm{e}^{-} / \mathrm{s}$.

The main noise sources are the stellar leakage, the detector noise, the thermal noise of the optics and the shot noise. The exo-zodiacal light in 2.5-5 $\mu \mathrm{m}$ is negligible. The local zodiacal light was taken into account according Leinhart et al, $1998^{9}$ but has a negligible effect in our case because it is supposed constant and is less than $1 \mathrm{e}^{-} / \mathrm{s}$. Our preliminary analysis does not consider any stray light from the sun, the moon, the earth or the deep sky.

As the nulling is not perfect, some star light reaches the detector, resulting in a stellar leakage: 


$$
I_{*}=\pi \eta_{o}(\lambda) \eta_{q}(\lambda) \eta_{*}(\lambda, B) A\left(\frac{R_{*}}{d}\right)^{2} B_{\lambda}\left(T_{*}\right) \Delta \lambda
$$

$\eta *(\lambda, B)$ is the residual star transmission of the interferometer. Its minimum value depends on the star diameter $\Delta \theta *$ which is partly resolved by the interferometer. It is also impacted by the instrument defaults like the intensity mismatch $\left(\mathrm{I}_{2} / \mathrm{I}_{1}=1+\varepsilon\right)$ and the residual OPD $(\mathrm{x}=2 \pi \delta / \lambda)$. In a first approximation we have:

$$
\eta_{*}(\lambda, B)=\left(1+\frac{\varepsilon}{2}\right)-\sqrt{1+\varepsilon} \cos (x)\left(1-\frac{X^{2}}{8}+\frac{X^{4}}{192}\right)
$$

Assuming statistics on $\varepsilon$ and $\delta\left(<\varepsilon>, \sigma_{\varepsilon},<\delta>, \sigma_{\delta}\right)$, one can derive the statistics on $\mathrm{I}_{*}\left(<\mathrm{I}_{*}>, \sigma_{*}\right)$ using Monte-Carlo

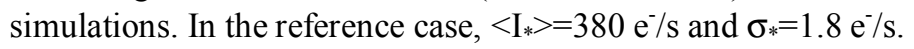

Optics are considered as grey bodies with emissivity $\varepsilon(\lambda)$ and temperature $<\mathrm{T}_{0}>$ with stability $\sigma_{\mathrm{TO}}$. The corresponding flux on the detector can be estimated by:

$$
I_{t h}=\pi A\left(\frac{a \lambda}{D}\right)^{2} \int_{\lambda-\Delta \lambda / 2}^{\lambda+\Delta \lambda / 2} \eta_{q}(\lambda) \varepsilon(\lambda) B_{\lambda}\left(T_{o}\right) d \lambda
$$

The beam etendue is limited to a fraction of the Airy radius: a $1.22 \lambda / \mathrm{D}(\mathrm{a}<1)$. In the reference case $\mathrm{I}_{\mathrm{th}}$ is completely negligible. At $5 \mu \mathrm{m},<\mathrm{I}_{\mathrm{th}}>=0.9 \mathrm{e}^{-} / \mathrm{s}$ and $\sigma_{\mathrm{th}}=0.3 \mathrm{e}^{-} / \mathrm{s}$ whereas $<\mathrm{I}_{\mathrm{p}}>=10 \mathrm{e}^{-} / \mathrm{s}$.

The detector noise consists in a read out noise $\left(\sigma_{\mathrm{RON}}\right)$ and a dark current variation $\left(\sigma_{\text {dark }}\right)$ coming from the thermal stability of the detection plane $\left(<\mathrm{T}_{\mathrm{d}}>\right.$ and $\left.\sigma_{\mathrm{Td}}\right)$. A numerical fit of data for $\mathrm{Hg}$-Cd-Te detector assuming a $5 \mu \mathrm{m}$ upper limit and a $18 \mu \mathrm{m}$ pixel leads to the following law : $\mathrm{I}_{\text {dark }}=1.6310^{-35} \mathrm{~T}_{\mathrm{d}}{ }^{19.6} \mathrm{e}^{-/ \mathrm{s} / \mathrm{pixel}}$.

For a total integration time $\tau_{\mathrm{i}}$, the shot noise is $\sigma_{\text {shot }}=\sqrt{\tau_{i} \sum I_{k}}$ and the SNR in nulling mode writes:

$$
S N R=\frac{1}{\sqrt{2}} \frac{I_{p} \tau_{i}}{\sqrt{\sigma_{\text {shot }}{ }^{2}+f \tau_{i} \sigma_{R O N}{ }^{2}+\left(\sigma_{*}{ }^{2}+{\sigma_{t h}}^{2}+\sigma_{\text {dark }}{ }^{2}\right) \tau_{i}^{2}}}
$$

Some results are illustrated by figure 5 which shows the various components of the SNR as function of $\lambda$ with the nominal set of parameters. In the lower part of the wavelength range, the SNR is limited by the nulling stability. It is directly linked to the stability and mean value of the OPD and of the intensity mismatch. Achieving the desired SNR below $2.5 \mu \mathrm{m}$ would mean a sub nm OPD control what would lead to a much more complex mission. As a compromise between the scientific interest and the mission cost, the lower wavelength limit was set to $2.5 \mu \mathrm{m}$ which still allows the detection of $\mathrm{CH}_{4}, \mathrm{H}_{2} \mathrm{O}$ and $\mathrm{CO}$. In the upper part of the spectrum, the performance is limited by the temperature of the optics and its stability. Again, a system trade-off was performed and reduced the upper limit from $6 \mu \mathrm{m}$ (initial proposal) to $5 \mu \mathrm{m}$ to avoid a too complex thermal design. In the middle part, we set the integration time and the detector temperature so that the photon noise and the detector noise only limit the performance for targets farther than $100 \mathrm{pc}$. The resulting optimized set of parameters is listed in the first column of table 1. Seven objects of the initial list have SNR $>10$ in the whole spectral range. Five other hot Jupiters have a medium difficulty level with $5<\mathrm{SNR}<10$. Four objects have SNR $<5$ and are considered too difficult for the Pegase mission.

Similar calculations were performed for the brown dwarfs and for the $\mathrm{V}^{2}$ mode applied to gap detection in the disks. The $\mathrm{V}^{2}$ mode can also be applied to hot Jupiters spectral characterization by analysing very small oscillations $(0.001$ order of magnitude) in the visibility curve and is then an alternative to nulling. The associated constraints on the system parameters are listed in table 1 . The hot Jupiters are the most demanding case for the mission, with a very stringent requirement on the stability of the OPD at $2.5 \mathrm{~nm}$ rms level. The intensity mismatch translates into a 30 mas pointing stability (see section 4 and 5) which is also quite demanding. A $0.1 \mathrm{~K}$ temperature stability of the focal plane is required to avoid too important dark current variations. The temperature of the optical bench shall not exceed $100 \mathrm{~K}$ with $1 \mathrm{~K}$ stability. The $\mathrm{V}^{2}$ and nulling modes lead to comparable constraints although the $\mathrm{V}^{2}$ mode slightly relaxes the temperature of the detector and the pointing, but at the price of a longer integration time and longer baselines. The $\mathrm{V}^{2}$ mode for the detection of gaps in disks is clearly a less demanding objective. 

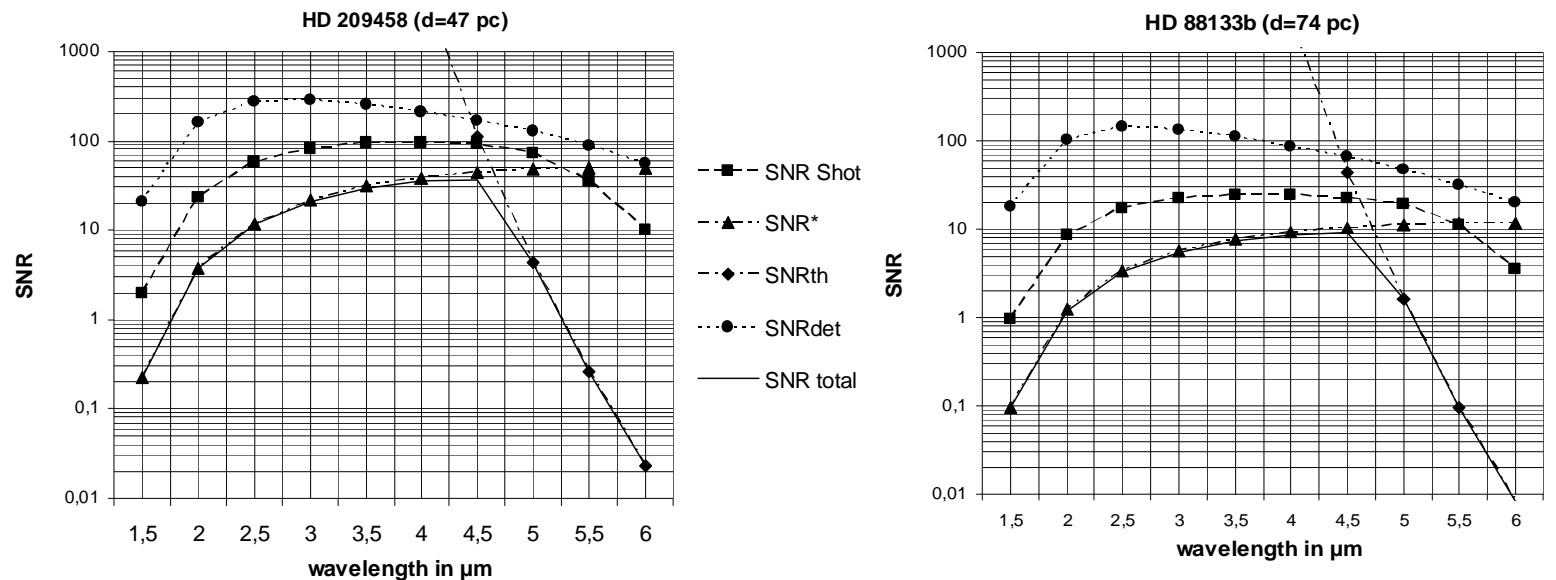

Figure 5: SNR calculation in nulling mode. Left : easy target . Right : moderately difficult target

Nominal parameters : $\tau_{\mathrm{i}}=10 \mathrm{~h}, \mathrm{~T}_{\mathrm{p}}=1400 \mathrm{~K}, \mathrm{R}_{\mathrm{p}}=1.5, \mathrm{~S}_{\mathrm{p}}=1, \mathrm{a}=1, \mathrm{D}=40 \mathrm{~cm}, \mathrm{R}=60, \eta_{\mathrm{o}}=0.07, \eta_{\mathrm{q}}=0.6,<\mathrm{T}_{\mathrm{d}}>=55 \mathrm{~K}, \sigma_{\mathrm{Td}}=0.1 \mathrm{~K} \mathrm{rms}$, $<\mathrm{T}_{\mathrm{o}}>=100 \mathrm{~K}, \sigma_{\mathrm{To}}=1 \mathrm{~K} \mathrm{rms},<2 \pi \delta / \lambda><510^{-3} \mathrm{rad},<\varepsilon><0.01, \sigma_{\delta}=2.5 \mathrm{~nm} \mathrm{rms}, \sigma_{\varepsilon}=0.003 \mathrm{rms}, \sigma_{\mathrm{RON}}=10 \mathrm{e}^{-} \mathrm{rms}$

\begin{tabular}{|c|c|c|c|c|}
\hline Parameter & $\begin{array}{l}\text { Hot Jupiters } \\
\text { nulling mode }\end{array}$ & $\begin{array}{l}\text { Brown dwarfs } \\
\text { nulling mode }\end{array}$ & $\begin{array}{l}\text { Gap in disks } \\
\mathbf{V}^{2} \text { mode }^{(2)}\end{array}$ & $\begin{array}{l}\text { Hot Jupiters } \\
\mathbf{V}^{2} \text { mode }^{(3)}\end{array}$ \\
\hline 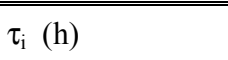 & 10 & 10 & 3 & 30 \\
\hline $\mathrm{B} / 2(\mathrm{~m})$ & $50-200$ & $5-80$ & $50-250$ & $100-400$ \\
\hline$\sigma_{\delta}(\mathrm{nm} \mathrm{rms})$ & 2.5 & 2.5 & 15 & 2.5 \\
\hline$<\mathrm{x}>(\mathrm{rad})$ & 0.001 & 0.001 & 0.02 & 0.005 \\
\hline $\begin{array}{l}\sigma_{\varepsilon}(\mathrm{rms}) \\
\text { (pointing) }\end{array}$ & $\begin{array}{c}0.003 \\
\text { (30 mas) }\end{array}$ & $\begin{array}{c}0.003 \\
\text { (30 mas) }\end{array}$ & $\begin{array}{c}0.03 \\
(300 \mathrm{mas})\end{array}$ & $\begin{array}{c}0.005 \\
(50 \mathrm{mas})\end{array}$ \\
\hline$<\varepsilon>$ & 0.01 & 0.01 & 0.1 & 0.01 \\
\hline$\sigma_{\mathrm{To}}(\mathrm{K} \mathrm{rms})$ & 1 & 1 & 1 & 1 \\
\hline$<\mathrm{To}>(\mathrm{K})$ & 100 & 100 & 105 & 100 \\
\hline$\sigma_{\mathrm{Td}}(\mathrm{K} \mathrm{rms})$ & 0.1 & 0.1 & 0.1 & 0.1 \\
\hline$<\mathrm{T}_{\mathrm{d}}>(\mathrm{K})$ & 55 & 55 & 60 & 60 \\
\hline RON (e- rms) & 10 & 10 & 50 & 50 \\
\hline Nbr of targets ${ }^{(5)}$ & 12 & 3 & $>100$ & 12 \\
\hline
\end{tabular}

Table 1 : driving parameters in various modes

${ }^{(1)} \mathrm{SNR}>10$ in $2.5-5 \mu \mathrm{m}$ for easy targets, ${ }^{(2)} \mathrm{K}_{\mathrm{lim}}=10, \mathrm{SNR} \mathrm{V}^{2}=200,{ }^{(3)} \mathrm{SNR} \mathrm{V}^{2}=2000$ at $5 \mu \mathrm{m} ; 10000$ at $2.5 \mu \mathrm{m}$

(4) intensity mismatch constrains the pointing through fiber coupling, ${ }^{(5)}$ in 2005

\section{4 - FORMATION FLYING CONTROL}

In our study we did not consider the deployment phase just after the injection on the orbit. We focused on the last control phase assuming the formation has been previously put in a coarse situation after a manoeuvre or a recovery from a safe mode. The initial state is given by the pointing and positioning of the spacecrafts obtained with standard star trackers (ST) and radiofrequency metrology (RF).

\section{1 - General principles}

Trying to match the pointing and OPD stability requirements from table 1 with a one level control architecture based on continuous actuation at satellite level only means using micro-thrusters and sensors with ultra-high accuracy. The 
associated technologies are very complex. To reduce costs and risks, we oriented our effort toward more conventional technologies such as impulsive cold gas thrusters and tried to relax the requirements on the spacecrafts attitude and position control. This led us to consider a two level control architecture combining control loops operating at satellite level and fine control loops implemented inside the optical payload. These fine loops have to compensate the external residual movements by corresponding displacements of optical delay lines (ODL) and fast steering mirrors (FSM). They must have a high enough bandpass to reject noises with sufficient efficiency. The limitations of such systems come mainly from undesired high frequency noise such as microvibrations from small mobile parts amplified by structural resonances as already studied in the SIM case for example (Miller, 2001 ${ }^{10}$ ). A good compromise between the two control levels is a central issue for the feasibility of the mission.

As the internal mechanisms have a finite stroke, they can not compensate the spacecrafts movements during the integration time specified in table 1 . It has to be split into elementary integration periods during which the vehicles are left drifting under the natural perturbations. Small impulsive manoeuvres are applied between two quiet periods to avoid the saturation of the fine mechanisms. Some relaxation in terms of metrology accuracy becomes possible since the absence of thrusters noise allows the improvement of estimation performances. The corrective manoeuvres will create an OPD shift exceeding the $2.5 \mathrm{~nm}$ level and interrupting the scientific observation for a short time (loss of the fine fringe tracking but the OPD remains in the coherence length). The key point is therefore the duration of the quiet periods and the scientists have agreed so far to split the observations in $100 \mathrm{~s}$ slices.

As the external disturbances introduced by the absolute positioning of the baseline with respect to the observed astronomical object can only be measured from the light emitted by the object itself, the fine correction stage has to be based on the light emitted by the star around which orbits the weak companion of interest. Maximizing the flux on the fine sensors is of critical importance for the ultimate nulling performance. The beam diameter and the total transmission up to their detection plane shall be maximized. The fine sensors are thus included in the optical payload and use part of the flux collected by the beam compressors. The estimated performances, computed in the two following subsections for piston and tip/tilt, show that the best spectral domain is the visible. This is an important result since efficient spacequalified detectors are available in the visible and this saves all the IR photons for the scientific instrument. A similar scheme is used on most ground-based adaptive optics systems.

\section{2 - Fine pointing internal loop}

Starting with the residual rotations of the spacecrafts, the fine pointing loop has to ensure a symmetrical coupling into the fiber as specified in table 1 and a minimal loss of flux (transmission $>0.7$ from 2.5 to $5 \mu \mathrm{m}$ ). The focal length of the injection parabola is adjusted to optimize the coupling at $2.5 \mu \mathrm{m}$. Assuming that the coupling efficiency follows the Strehl approximation (Ruilier, $2001^{8}$ ) and a mean intensity mismatch of $10^{-2}$, a specification of around 30 mas per axis for the pointing accuracy has been evaluated.

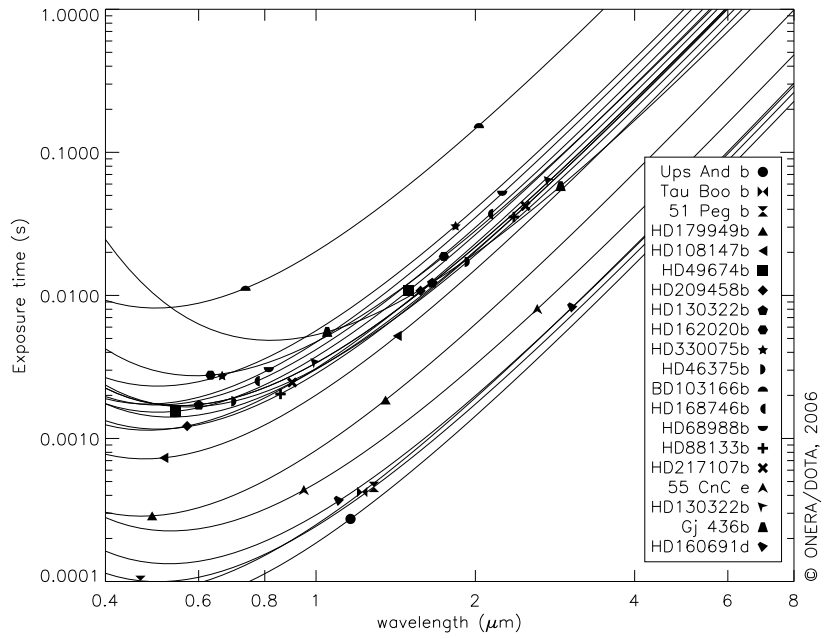

Figure 6 : fine pointing integration time vs wavelength
The baseline approach to measure the tip-tilt errors consists in using the visible-near infrared domain where the stellar spectrum reaches its maximal values for most stars. The estimation of the signal error will be made with images recorded by a camera common to the two arms and located in an image plane: the field relative angle sensor (FRAS). An optimized algorithm will be used to minimize the contribution of the photon noise.

The tip/tilt error will be controlled in a feedback loop thanks to two fast FSM based on piezoelectric actuators. Since the FSM are located in the compressed beam, the optical gain M of the M2-M3 beam compressor (see section 5) will be a trade-off between dynamics of the tip-tilt errors (dynamical disturbances and drift effects due to various sources like alignment errors, thermal dilatation or gravity release), noise and stroke actuation and mechanical constraints. $\mathrm{M}=20$ is today considered but current 
work tends to lower this value to relax the inertial pointing control of the combiner. Regular calibration procedures are foreseen to maximize the coupling efficiency into the fibers and to measure the interaction matrix between the FSM voltages and the spot position in the camera plane.

The exposure time required to reach a 10 mas contribution for the photon noise has been computed for the target stars. Figure 6 plots the estimated exposure time versus the wavelength assuming a spectral bandwidth $R_{\lambda}=\lambda / \Delta \lambda$ of 2.5 and a total transmission of 0.11 in the considered spectral domain. It appears that the exposure time can be smaller than a few ms for most of the stars which means that the contribution of the photon noise is not dominant. The main task should be to correct the disturbances due to instrumental vibrations and control attitude errors.

\section{3. - Fine OPD control}

The fine OPD-control loop is made of two main components: the ODL already mentioned and the Fringe Sensor (FS) used to measure the OPD between the beams.

In the observing mode, the main requirement is that the residual OPD should be less than $2.5 \mathrm{~nm}$ rms. The exact performance can not be estimated for the moment, since the amplitude of most disturbances is not known at the nanometer level. What can be done is to suppose that the FS and the ODL are run at the maximum frequency to sufficiently damp all the OPD contributors, so that the residual OPD mostly comes from the measurement noise of the FS and is equal to $2.5 \mathrm{~nm}$ rms. Since the FS noise is directly linked to the number of detected photons $\left(\right.$ Cassaing, $\left.2001^{11}\right)$ and since the magnitude of all the targets is known, an estimate of the FS integration time can be derived as plotted in figure 7a. This figure confirms that the best spectral domain for the FS is the visible. An integration time of about $20 \mathrm{~ms}$ is sufficient for most targets, i. e. a correction frequency from around $50 \mathrm{~Hz}$ up to $200 \mathrm{~Hz}$ for a few targets. This should be sufficient to control OPD contributors at low frequency (residual speed, differential solar pressure,...) or at high frequency (microvibrations) since their amplitude is smaller and advanced control techniques (such as Kalman filtering) can be used for known disturbances (constant acceleration, sinusoidal harmonics). To optimize the measurement, a rather large spectral band $\left(\mathrm{R}_{\lambda}=\lambda / \Delta=2.5\right)$ and a small number of pixels (photon-noise regime) have been assumed.
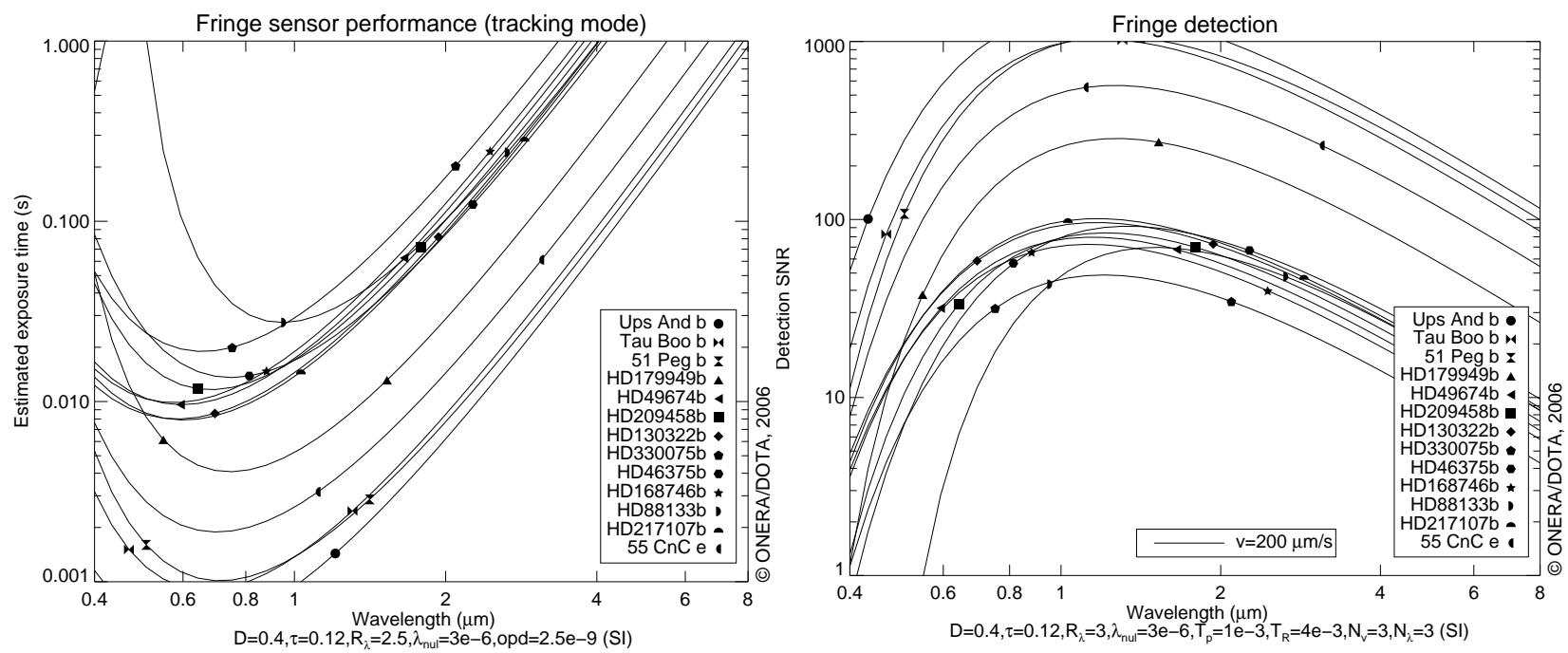

Figure 7: Estimation of the integration time required in the FS to reach an OPD accuracy of $2.5 \mathrm{~nm}$ (a, left) and SNR for fringe detection in the acquisition mode (b, right).

In the acquisition mode, the OPD between the beams can be much larger than the coherence length of the FS. It is thus necessary to perform a fringe search, by moving the ODL or waiting for the fringes to pass while the spacecrafts drift. Because of the size of the baseline, the speed $v$ of the fringes can reach several hundreds of $\mu \mathrm{m} / \mathrm{s}$. To avoid blurring, the exposure time $T_{p}$ of the FS must therefore be close to $1 \mathrm{~ms}$, and the repetition time $T_{r}$ small enough so that measurements at locations $v T_{r}$ correctly sample ( $N_{v}$ times) the chromatic envelope of the fringe pattern, which coherence length is given by the number of spectral channels $N_{\lambda}$. Figure $7 \mathrm{~b}$ shows that for detection, the best spectral domain is shifted towards the IR, because the long wavelengths less suffer from blurring. However, for the targets of interest, a SNR larger than 10 is possible in the visible using realistic parameters for the real-time processor and an external OPD drift up to $200 \mu \mathrm{m} / \mathrm{s}$. 
The FS must meet requirements from the acquisition and tracking modes. In order to reach the kHz sampling rate with a minimum number of pixels, a coaxial beam combiner is the most relevant to combine the two beams. To cope with stability requirements and the fast fringe drift, a spatial modulation is used without any moving part. Such a FS has already been investigated in our team for stellar interferometry on ground (Cassaing, 2000 ${ }^{12}$ ). Light from each of the four $\pi / 2$ phase-shifted output is dispersed and focused on a linear array of pixels. Using all the available visible range $(\lambda$ between 0.6 and $0.9 \mu \mathrm{m}$, cut in 3 spectral channels of $100 \mathrm{~nm}$ ) gives a spectral resolution $R_{\lambda}=2.5$.

\section{4 - Satellite control}

Environment perturbations: In L2 environment, the dominant disturbances come from the solar radiation pressure that produces differential forces and torques due to non identical surface to mass ratios and lever arms between the center of pressure and the center of mass. Using the dimensions and the mass properties coming from the pre-design, it has be shown that the attitude bounds will be exceeded much faster than position bounds in absence of control: a $5 \mathrm{~mm}$ drift occurs after $1000 \mathrm{~s}$ whereas a 10 "' pointing error is reached after $100 \mathrm{~s}$ in the worst case with $\beta=30^{\circ}$.

Hybrid architecture: The impulsive control approach leads to the problem of synchronising the gas pulses. The high number of degrees of freedom (6 for relative positioning, 18 for attitude) combined with their associated dynamics would require a complex synchronization algorithm so as to ensure the $100 \mathrm{~s}$ quiet period. To avoid this complexity we chose an hybrid architecture based on reaction wheels to ensure pointing requirements of the siderostats. The pointing of the combiner and the relative positioning are still performed with cold gas systems. A preliminary analysis shows that the microvibrations generated by the reaction wheels on the siderostats are consistent with the requirements on pointing and OPD stability. The stiff structure of the siderostats and the light reaction wheel are favourable factors. Actual studies are focusing on this matter and on the torque fluctuations which need to be further investigated.

Platforms control objectives: The first issue for the nominal mode control is to keep the internal mechanisms within their working domains during the elementary integration of $100 \mathrm{~s}$. This requires that the external OPD stays below the assumed delay line stroke. In parallel the cumulated pointing errors of the combiner and the siderostat must ensure that the beam deviations remain within the FSM stroke. As the use of the FRAS and reaction wheels will allow a relative pointing control of the siderostats at 1" accuracy level, we found that the inertial pointing of the combiner can be relaxed to +/- 5 to 10 ", depending on the exact value of the angular magnification (M), the FSM stroke and its allocation between the attitude corrections and the optical misalignment compensation.

Another key function of the control is to lead the formation from a deployment state to a fine stabilized state. This final state requires that the external OPD velocity is less than 100-150 $\mu \mathrm{m} / \mathrm{s}$ (with some margins w.r.t. section 4.3 result). Such a requirement applies to the cumulated effects of various degrees of freedom (DOF) both in translation and rotation. An allocation is needed between the various terms. As a preliminary rule, we specified a equivalent velocity control accuracy of 10 to $20 \mu \mathrm{m} / \mathrm{s}$ rms on each DOF of interest.

Calibration process: As the initial cumulated bias of the various DOF metrologies used to estimate the external OPD will exceed the stroke of the ODL, a calibration process is needed. For the first fringe acquisition, and later at regular intervals during the mission, it is foreseen to scan the OPD using the movement of one siderostat in order to estimate this cumulated bias with a precision roughly equal to the coherence length of the science interferograms (about $100 \mu \mathrm{m})$. Consequently, there is no need for a very accurate distance sensor (sub mm level) to reduce the total initial OPD bias.

Filtering: Filtering algorithms using the predictable movement laws in L2 environment will be used to relax the resolution of the sensors imposed by the formation damping requirement. A preliminary work performed at ONERA under CNES R \& D contract estimates that a factor 10 to 100 can be saved this way.

Sensors specifications: the metrology was defined to fulfil the positioning and stability requirements with the use of filtering and initial calibration of the OPD uncertainty. For the translation DOF, biases of $1 \mathrm{~cm}$ are acceptable on the absolute position measured by the sensors (RF metrology along the X axis, 10" bias on lateral sensor). The relative displacements must be measured with $0.1 \mathrm{~mm}$ to $1 \mathrm{~mm}$ resolution at $10 \mathrm{~Hz}$, depending on the exact filtering performance. Along the $\mathrm{X}$ axis, this may be achieved by the RF metrology in an optimistic case but to be more conservative, the nominal set up should include a simple laser telemeter (resolution of 1 fringe is sufficient). Along the other axes, the lateral sensor should have a 0.1 to 1 arcsec resolution at $250 \mathrm{~m}$. According to a first study performed by the French company Sodern under CNES R \& D contract, this is possible using small cameras on the combiner and a laser diode based illuminator on each siderostat $(\lambda=850 \mathrm{~nm})$. As far as pointing is concerned, a fine star tracker of the arcsec class is very probably needed in the payload of the combiner to fulfil the damping and pointing requirements. 
Propulsion specification: The analysis of the non continuous control shows that classical cold gas thrusters with a minimum impulse bit (MIB) of 0.05 m.N.s are needed.

Sensors and modes sequence: After deployment in L2, the formation keeping relies on the RF metrology stage that provides an instantaneous accuracy of $1 \mathrm{~cm}$ in distance and of $1^{\circ}$ in lateral position (half angle cone, a few meters at $250 \mathrm{~m}$ ). The residual velocity is $5 \mathrm{~cm} / \mathrm{s}$. The pointing accuracy is about 10" for each spacecraft obtained with standard $\mathrm{ST}$. The coarse lateral sensor $\left(\mathrm{FOV}\right.$ of $3^{\circ}$ ) reduces the lateral error down to the arcsec level (a few $\mathrm{mm}$ at $250 \mathrm{~m}+$ alignment bias) w.r.t to the combiner frame. The next step consists in acquiring the guide star in each FRAS and in the fine ST. The FOV of the FRAS is 90 " for each arm and cover the cumulated pointing errors achieved by each satellite with a standard ST including some margins. The information coming from the FRAS and the fine ST are used to improve the combiner inertial pointing and the relative pointing of the siderostat and to damp associated movements. In parallel, the lateral position offset is reduced to allow the longitudinal sensor acquisition and translation motions are stabilized to less than $10-20 \mu \mathrm{m} / \mathrm{s}$ by relying on the global estimator.

\begin{tabular}{|c|c|c|c|c|}
\hline $\begin{array}{c}\text { RF sensor } \\
\text { standard STR }\end{array}$ & $\begin{array}{l}\text { Coarse Position } \\
\text { \& pointing control }\end{array}$ & $\begin{array}{l}\text { Coarse lateral } \\
\text { sensor acquisition }\end{array}$ & & \\
\hline $\begin{array}{c}\text { Coarse Lateral } \\
\text { sensor }\end{array}$ & & $\begin{array}{l}\text { improvement of } \\
\text { position control }\end{array}$ & $\begin{array}{l}\text { FRAS and fine } \\
\text { STR acquisition }\end{array}$ & \\
\hline & & & $\begin{array}{l}\text { Improvement of } \\
\text { attitude control }\end{array}$ & $\begin{array}{c}\text { Acquisition of fine } \\
\text { metrology }\end{array}$ \\
\hline $\begin{array}{c}\text { Fine } \\
\text { longitudinal } \\
\text { sensor }\end{array}$ & & & & $\begin{array}{l}\text { Improvement of } \\
\text { drift along } X \text { axis }\end{array}$ \\
\hline $\begin{array}{l}\text { Measurement } \\
\text { precision }\end{array}$ & $\begin{array}{l}\mathrm{X} \text { position : } 1 \mathrm{~cm} \\
\mathrm{Y}, \mathrm{Z}: 1^{\circ} \text { half cone } \\
\text { pointing } 10^{\prime \prime}\end{array}$ & $\begin{array}{c}\mathrm{Y}, \mathrm{Z} \text { position : } 1.25 \mathrm{~mm} \\
\text { lateral @250 m } \\
\text { velocity : few } 10 \mu \mathrm{m} / \mathrm{s}\end{array}$ & $\begin{array}{c}X, Y, Z \text { angles : few " } \\
\text { velocity : a few } 0.01 " / s\end{array}$ & $\begin{array}{c}\Delta \mathrm{X} \text { velocity : } 10 \mu \mathrm{m} / \mathrm{s} \\
\mathrm{ddm}<+/-1 \mathrm{~cm} \\
\mathrm{~d}(\mathrm{ddm}) / \mathrm{dt}<150 \mu \mathrm{m} / \mathrm{s}\end{array}$ \\
\hline
\end{tabular}

Figure 8: sequencing of the metrologies

\section{5 - OVERVIEW OF THE PAYLOAD}

A possible optical layout is illustrated by figure 9. Many constraints apply on the design. We first had to cope with a limited volume $(120 \times 120 \times 60 \mathrm{~cm})$ because of the system choice to fit into a Soyuz fairing without deployable sun shields (see 6.4). Second, the reliability in space environment imposes the redundancy of all mechanisms. Then, we minimized the number of components in order to reduce the complexity and maximize the optical transmission. Another guideline was to minimize the coupling of various degrees of freedom, either at satellite level or in the payload itself. That's why we imposed the stop on FSM so that the tip/tilt correction does not affect the OPD. Last, the two arms of the interferometer shall have a maximum symmetry w.r.t. reflections to minimize the differential polarization effects.

Siderostat: M1 is a flat mirror with $45^{\circ}$ inclination and an elliptical aperture. Its size $(70 \times 50 \mathrm{~cm})$ is constrained by the relative positioning of the spacecrafts.

Afocal: $M 2 / M 3$ form a simple afocal with a magnification $M=20$. An off axis afocal could also be implemented but it leads to a smaller collecting diameter and a larger optical bench assuming f/D $>1$.

Achromatic phase shifter: M4 is a small flat mirror which directs the rays towards the active stage. Combined with the M1 layout, it creates an achromatic $\pi$ phase shifter using field reversal by reflections (Serabyn, $1999^{13}$ ). Although this solution seems very elegant, one may not exclude the need to compensate small chromatic effects by dispersive prisms like the ones used by the Synapse or MAII breadboard (Brachet, $2005^{14}$, Weber $2004^{15}$ ). 
Active stage: M5 and M6 form a cat's eye type ODL. We assumed a $+/-1 \mathrm{~cm}$ stroke and $1 \mathrm{~nm}$ rms resolution is feasible with magnetic bearings and voice coil actuation (Van den Dool, 2004 ${ }^{16}$ ). The ODL is placed before the FSM and the curvature of its secondary mirror is adjusted to image the pupil on the FSM backwards near M3. Each FSM uses three piezoelectric actuators with $15 \mu \mathrm{m}$ stroke and nm resolution assuming a $100 \mathrm{~V}$ maximum voltage and a $5 \mathrm{mV}$ electronic noise. The angular stroke is $+/-65^{\prime}$ including a $35 \%$ cut off for operation at $100 \mathrm{~K}$. The first estimates of differential polarization effects induced by the $45^{\circ}+/-65^{\prime}$ angle shows that the impact on nulling performance is less than $10^{-8}$.

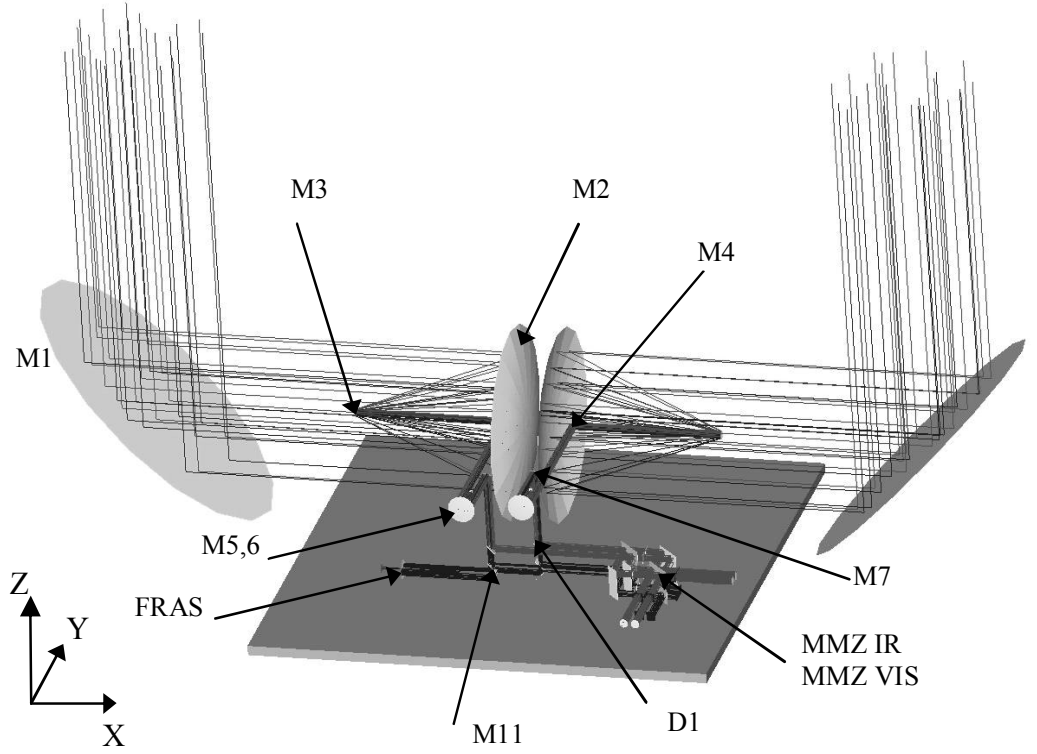

Figure 9: preliminary optical layout

Combining stage: A dichroïc plate transmits the wavelength below 1.5 $\mu \mathrm{m}$ and reflects higher wavelengths toward a modified Mach-Zender performing a full symmetric and achromatic recombination for the nulled outputs (Serabyn, 2001 ${ }^{17}$ ). Small off axis parabolas focus the four outputs of the MMZ into single mode fibers made of fluorid glasses $\left(\lambda_{\mathrm{c}}=2.5 \mu \mathrm{m}, \quad \mathrm{NA}=0.15\right.$, $\mathrm{r}_{\mathrm{c}}=6.32 \mu \mathrm{m}, \mathrm{ZrF}_{4}$ based glasses, Mitachi, $1983^{18}$, Mazé, $1984^{19}$ ).

Detection module: These fibers are connected to a detection stage including a dispersing prism and a $\mathrm{Hg}-\mathrm{Cd}-\mathrm{Te}$ based detection matrix. It is not represented on figure 9 but will lie toward the $-\mathrm{Y}$ side of the bench, with a high thermal decoupling and a link to a sufficient radiator on the $+Z$ face.

Internal loops sensors: The visible part of the spectrum is divided between the FS and the FRAS. The exact allocation is still to be optimized. The FS is another MMZ with the four classical ABCD outputs generated by a spatial modulation. Both MMZ are superposed and the differential OPD is minimized to avoid stability problems such as the ones detected in the recent nulling breadboards (Brachet, 2005 ${ }^{14}$ ). The FRAS is based on a Cassegrain type small telescope with a classical 1024 x $1024 \mathrm{Si}$ based CCD where 1 pixel represents 0.18 " on the sky. Each beam uses a different 512 x 512 sub-matrix thanks to a small differential tilt angle of M11 mirrors. The estimated resolution of the camera is 10 mas.

The $+Y$ part of the bench will be used to implement other metrology sensors required by the GNC (see section 4.4): a precise star tracker $(1$ arcsec) and possibly a laser metrology system that could be of the Mouse type (Le Duigou, $2005^{20}$ ). Electronics will be located outside the optical bench, in the service module, to avoid dissipation sources.

\begin{tabular}{|c|c|c|c|}
\hline Parameter & Value & Parameter & Value \\
\hline Mass & $70 \mathrm{~kg}$ & Mirror coatings & Gold, $\lambda_{\text {vis }} / 20$ \\
\hline Dimensions & $120 \times 120 \times 60 \mathrm{~cm}$ & Effective focal length & $1,800 \mathrm{~m}$ \\
\hline IR optical transmission ${ }^{(*)}$ & $7 \% 2.5-5 \mu \mathrm{m}$ & Optical bench Temperature & $100+/-1 \mathrm{~K}$ \\
\hline FRAS optical transmission ${ }^{(*)}$ & $12 \% 0.6-1.0 \mu \mathrm{m}$ & Detector temperature & $55+/-0.1 \mathrm{~K}$ \\
\hline SF optical transmission ${ }^{(*)}$ & $8 \% 0.6-1.0 \mu \mathrm{m}$ & Single mode fiber & fluorid glasses \\
\hline Collector diameter & $40 \mathrm{~cm}$ & Science Detectors & $\mathrm{Hg}-\mathrm{Cd}-\mathrm{Te}$ \\
\hline Magnification of afocal & $<20$ & FRAS resolution & $10 \mathrm{mas}$ \\
\hline
\end{tabular}

Table 2 : Main characteristics of the payload

${ }^{(*}$ includes beamsplitters and 0,7 fiber coupling, assumes 0,95 minimum reflectivity, does not include the quantum efficiency 


\section{6 - OVERVIEW OF THE SPACE SEGMENT}

\section{1 - Orbit selection}

A trade-off was performed between High Elliptical Orbits (HEO) and Sun-Earth system L2 point orbits (L2). Given all the constraints an eight days period orbit with a $40000 \mathrm{~km}$ high perigee and a $285000 \mathrm{~km}$ high apogee was selected in the HEO case. Although the gravity gradient is compatible with the mission on more than $90 \%$ of the orbit, it was not possible to prove at the stage of pre-design whether the variations of the heat flux due to Earth were compatible or not with the payload thermal stability needs (see 6.3). As the cost difference with the L2 option is not so significant, the HEO orbit was not chosen as the baseline for our study but the trade-off is not completely closed.

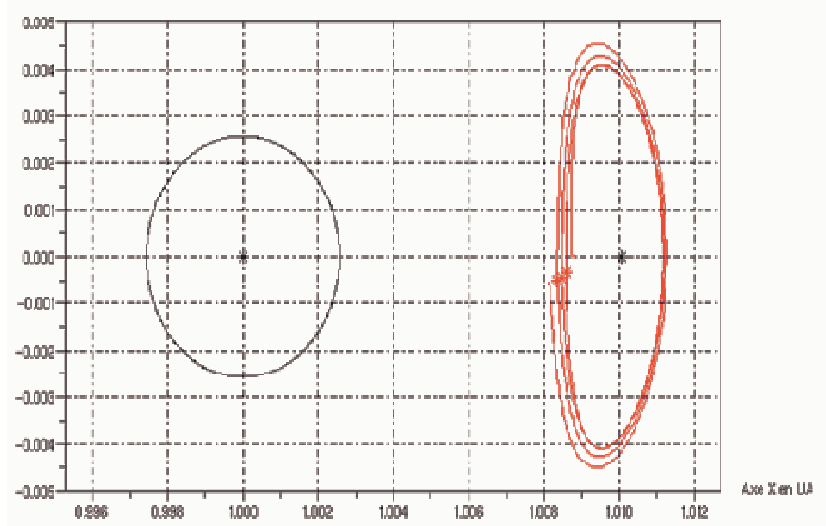

Figure 10: Chosen orbit (in black : Moon trajectory).

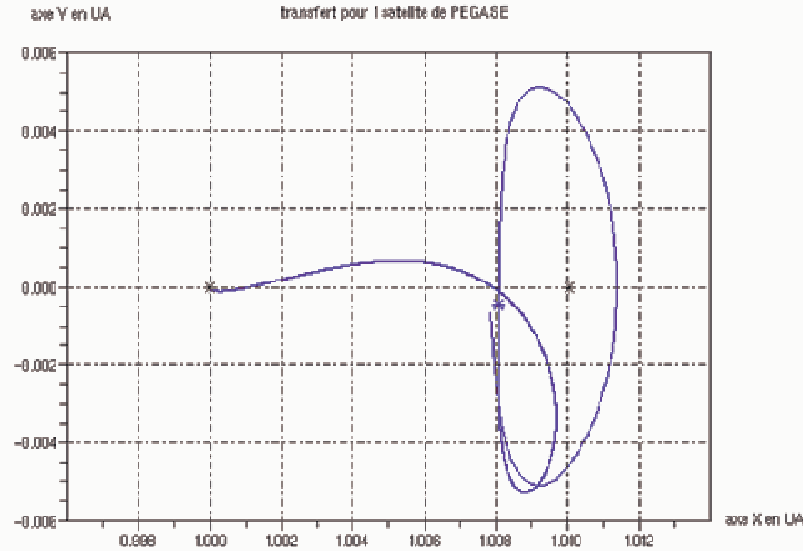

Figure 11: L2 transfer in the synodic

Two kinds of orbits have been investigated at L2: the halo and Lissajous orbits. Looking at the mission needs, there was no preference for one or the other. But on the topic of orbit maintenance the differences are quite significant: the Lissajous orbits are obtained thanks to linear analysis. For this reason they are much easier to operate, in particular with respect to the orbit determination performances related to the vehicles $\Delta \mathrm{V}$ manoeuvres precision realization. In addition the total $\Delta \mathrm{V}$ needed for the Lissajous is not more important then the one for a halo orbit. For both reasons a Lissajous orbit was retained in the study.

The chosen orbit minimizes the requested injection $\Delta \mathrm{V}$. Its parameters in the synodic plane are $250000 \mathrm{~km}(\mathrm{x}) / 673000$ $\mathrm{km}(\mathrm{y})$. In this plane it has an instable motion with a period of $\sim 177,6$ days. Out of this plane the motion is stable with a period of 184 days (see figure 10 and 11).

\section{2 - Operations and mission duration}

The critical operations are the rotation of the formation, the adjustment of the size of the baseline and the change of target. At L2 point, a typical telemetry and telecommand (TTC) session will last 8 hours. System wise it has been chosen
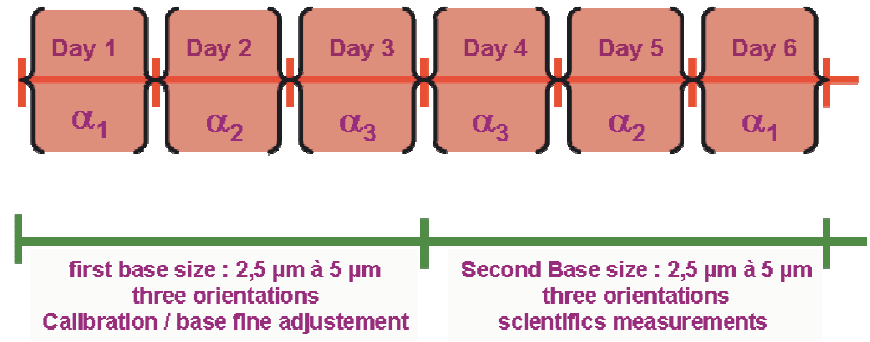

Figure 12: typical observation sequence (Hot Jupiters) to perform the critical operations under the monitoring of the ground segment. As a consequence during the TTC session the satellites will move to the next position with velocities of a few $\mathrm{cm} / \mathrm{s}$, download the telemetry and receive the flight program for the next day. A typical 6 days long sequence is illustrated on figure 12 in the nulling mode for hot Jupiters or brown dwarfs. Assuming 20 objects of each type, 240 days are needed to cover the scientific mission. Adding another 120 days for the observation of the disks, one gets a total scientific mission duration of one year. Considering a mean availability of the system of 0.75 , the total mission duration is estimated to 2.5 years, including a 4 months transfer phase, a 4 months commissioning phase and a 6 months margin for additional programs. 


\section{3 - Thermal control}

The system choice is to use a passive system to avoid the mechanical perturbations of the OPD by active cryogenic devices. Due to the earth albedo, maintaining the $0.1 \mathrm{~K}$ stability on the detector plane during a sufficient part of the 8 days HEO with a passive design seems difficult. The equivalent mean well temperature is $45 \mathrm{~K}$ as compared to $3 \mathrm{~K}$ at L2. The required radiator would be white painted with a $4.5 \mathrm{~m}^{2}$ surface to evacuate $1 \mathrm{~W}$. The required stability would impose a quite complex sun shield around the payload due to the various pointing configurations along the seasons and the orbit. Last, the formation would have to avoid the high perturbation peak near the perigee with a special de-pointing configuration. A detailed thermal analysis, which was out of the scope of our study, is needed to further investigate the feasibility of this option.

In the $\mathrm{L} 2$ case, the thermal control is based on a high decoupling between the service module $(300 \mathrm{~K})$ and the optical payload (100 K) using V-grooves systems. The decoupling of the detector stage $(55 \mathrm{~K})$ w.r.t. the optical bench is TBD. The radiator is dark painted and the surface to evacuate $1 \mathrm{~W}$ is only $2 \mathrm{~m}^{2}$. The V-grooves and the payload have to be protected from the direct sun light by sun shields assuming anti-solar pointing with a maximum depointing angle of $30^{\circ}$.

\section{4 -Launcher configuration}

After a detailed analysis, the use of existent platforms suggested in the initial CNES call for proposals proved to be inadequate for Pegase. The mechanical architecture is then based on dedicated platforms. To reduce the costs, the choice was made to use one single Soyuz launcher and to avoid deployable sun shields. The internal diameter of the launcher fairing becomes a major constraint. A compromise had to be made between the diameter of the collectors, the size of the vehicles, the allowable depointing angle $\beta$. We found that $\beta<30^{\circ}$ gives access to more than $90 \%$ of the identified scientific objects and is compatible with the Soyuz fairing with fixed sun shields if $\mathrm{D}<40 \mathrm{~cm}$ and a special structure is used to accommodate the three satellites under the Soyuz fairing. A solution based on the Sylda structure proved to be less efficient. The retained configuration is illustrated by figure 13. Figure 14 outlines the combiner.

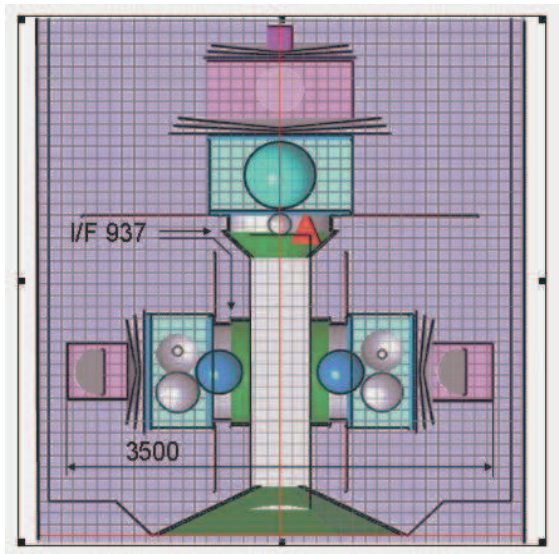

Figure 13: launch configuration

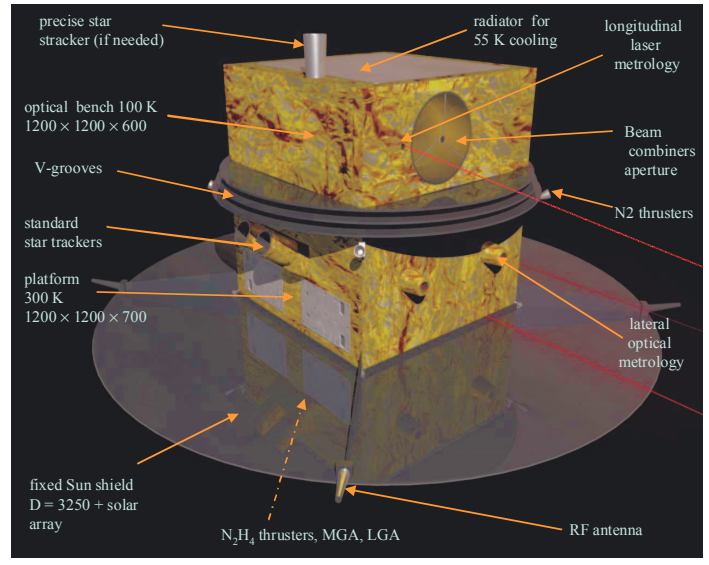

Figure 14: outline of the combiner satellite

\section{5 - Propulsion}

The nominal solution retained for the formation flying propulsion is an impulsive cold gas $\left(\mathrm{N}_{2}\right)$ system based on existing technology $(10 \mathrm{mN}$ thrusters from Marotta UK) with a MIB reduction down to $50 \mu \mathrm{N}$.s. That does not represent a strong technology push (a gain factor of 10 w.r.t. Grace thruster resolution) and seems affordable by reducing the minimal path of the thrusters divergent (today's diameter is $0.26 \mathrm{~mm}$ ), limiting the pulse duration and reducing the thrusters admission pressure (today it is roughly 1.3 bars for Marotta technology). The repeatability of the pulses would have to be studied in more details. Assuming $70 \%$ efficiency, the total $\Delta \mathrm{V}$ needed for the formation control (change of target, rotation and sizing of the formation), the fine orbit control and the failure detection and recovery system is $25 \mathrm{~m} / \mathrm{s}$ for the combiner and $17 \mathrm{~m} / \mathrm{s}$ for each siderostat. For high $\Delta \mathrm{V}$ (injection, rough positioning) the solution retained is a classical hydrazine system. The launcher corrections, the injection on the orbit and the orbital manoeuvres require about $92 \mathrm{~m} / \mathrm{s} \Delta \mathrm{V}$ on each spacecraft ( $98 \%$ efficiency). 


\section{6 - Telecommunication}

From a communication point of view, the formation is considered nominally as one spacecraft with a $600 \mathrm{Mbits} / \mathrm{day}$ total telemetry. The main link uses the X-band and $15 \mathrm{~m}$ ground antennas to communicate with the combiner equipped with 2 low gain antennas and 2 medium gain antennas. The data from the siderostats are collected through the intersatellite link which uses the RF metrology. Back-up links are available on the siderostats for the safe mode operations where independent access to each satellite is possible.

\section{7 - Mass and Power budgets}

The solar arrays are fixed and located at the back of the sun shields. Assuming $60 \%$ of AsGa3J solar cells, $40 \%$ of optical solar reflectors and a 0.7 fill factor, the available powers are $260 \mathrm{~W}$ for the siderostat and $770 \mathrm{~W}$ for the beam combiner which is largely sufficient to cover the payload and platforms needs. The mass budget was build upon a detailed analysis at component and sub-system levels using CNES internal databases. It also includes all liquid propellants. A 5 to $20 \%$ margin is applied at equipment level depending on the maturity and a global $30 \%$ system margin applies. The resulting mass estimates are $350 \mathrm{~kg}$ for each siderostat and $700 \mathrm{~kg}$ for the combiner. With the adapter and the interface structure, the total launch mass is estimated to $1785 \mathrm{~kg}$ which leaves a $13 \%$ margin w.r.t. the Soyuz performance $(2050 \mathrm{~kg})$.

\section{8 - Cost estimation}

At this stage, the cost estimation process of such a challenging mission is very difficult and subject to a lot of uncertainties. The difficulty is furthermore amplified by the free flying technology which is a major innovation without any prior mission as a reference. Rough order of magnitude (ROM) costs have been estimated thanks to an analytical method with appropriate margins depending on the technology maturity level. In addition, parallel costs databases for similar payloads and platforms have been used. As an example, the IASI interferometric payload has an equivalent final cost of $1 \mathrm{ME} / \mathrm{kg}$ which is very near from the figure obtained with the analytical method on the combiner payload. Assuming some other simpler mission validates the first level of formation flying, the total development plan (ABCD phases) is estimated to expand over a 8 to 9 years time period. Assuming a development mainly performed in the industry, the estimated ROM external cost is $320 \mathrm{ME}+/-20 \%$ (payload 70, launch 50, operations 20, combiner 110 , siderostats 70). An additional 10\% margin is taken to calculate the maximum cost. These figures are comparable with the estimations of NASA for similar missions such as FKSI (Danchi, 2004 ${ }^{21}$ ) or the early version of Starlight.

\section{7 - CONCLUSION}

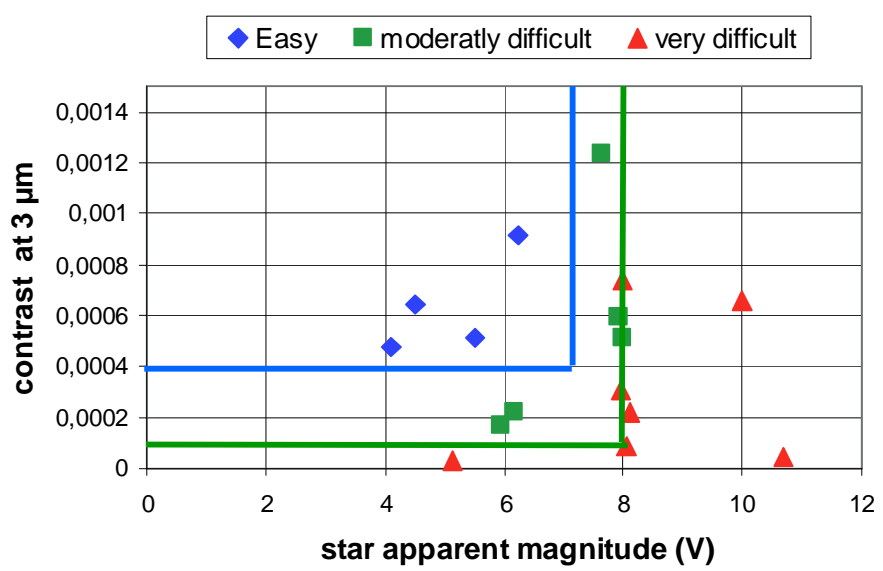

Figure 15 : performance domain of Pegase. Diamonds: easy HJ, squares: intermediate difficulty $\mathrm{HJ}$, triangles: impossible with current assumptions The OPD stability imposes a contrast better than $10^{4}$ and the FS requires central stars brighter than $\mathrm{V}=8$.

Figure 15 summarizes the estimated main performances of the Pegase mission for the spectroscopy in the nulling mode. Today, more than 12 objects ( $\mathrm{HJ}$ and $\mathrm{BD}$ ) can be found in this domain and more will come in the next years. With many possible other programs, such as the detection of gaps in protoplanetary disks, the expected scientific return is very valuable.

From an engineering point of view, this phase 0 study only gives the outlines of what could be the Pegase mission. Some major trade-offs are still open and many subjects remain of course to be investigated in much more details during a future phase A. Nevertheless, the resulting first picture is attractive and it seems to us that this mission could be a good compromise between the scientific interest and the technological challenge when placed into an international roadmap toward free flying interferometry in space. We therefore think that Pegase can be proposed to ESA/NASA as an intermediate pathfinder mission before TPF-I/Darwin if the planned funding for the next decade allows such an option. The future $\mathrm{R}$ and $\mathrm{D}$ work will focus on a breadboard of the payload including the nulling interferometer and the fine control loops. 


\section{REFERENCES}

1. Leyre X. et al., « First formation flying interferometric mission including on flight Bracewell nulling », $2^{\text {nd }}$ International Symposium on Formation Flying, Washington, sept. 2004

2. Sudarsky et al, « Theoretical Spectra and Atmospheres of Extrasolar Giant Planets », ApJ, Vol. 588, p 1121-1148, 2003

3. Barman T.S. et al, « Phase-Dependent Properties of Extrasolar Planet Atmospheres », ApJ, Volume 632, Issue 2, pp. 1132-1139, 2005

4. Bracewell R.N., « Detecting non solar planets by spinning infrared interferometer », Nature, 274, p.780, 1978

5. Léger A. et al., "Could we search for primitive life on extrasolar planets in the near future ? The Darwin project ", Icarus 123, 249-255, 1996

6. Angel J.R.P. and Woolf N.J., « An imaging nulling interferometer to study extrasolar planets », AJ, $475: 373,1997$

7. Ollivier M. and Mariotti J.M. «Improvement in the rejection rate of a nulling interferometer by spatial filtering », Applied Optics, Vol 36, $\mathrm{N}^{\circ}$ 22, p 5340-5346, 1997

8. Ruilier C. and Cassaing F. « Coupling of large telescope and single-mode waveguides » JOSA, Vol 18, $\mathrm{N}^{\circ} 1$, p 143-149, 2001

9. Ch. Leinert et al., « The 1997 reference for diffuse night sky brightness», A\&A SS, 127 1-99, 1998

10. Miller D.W. et al., « Integrated dynamics and controls modelling for the Space Interferometry Mission (SIM) », in IEEE Aerospace Conference, vol. 4, p2089-2102 2001

11. Cassaing, F., «Optical path difference sensors», Comptes-Rendus de l'Académie des Sciences, Série IV, tome 2, $\mathrm{n}^{\circ} 1$, pp. 87-98, Jan 2001.

12. Cassaing F., Fleury B., Coudrain C., Madec P.-Y., Di Folco E., Glindemann A. and Lévêque S., « An optimized fringe tracker for the VLTI/PRIMA instrument », proceedings of SPIE conference 4006 « Interferometry in optical astronomy », pp. 152-163, 2000.

13. Serabyn E. et al., Applied Optics, Vol. 38, No 34, pp 7128-7132, 1999

14. Brachet $\mathrm{F}$., « Etude et developpement d'un déphaseur achromatique pour l'interférométrie en frange noire » thèse de doctorat de l'université Paris-Sud (XI), 2005

15. Weber V., Barillot M. \& al. «Nulling interferometer based on an integrated optics combiner », SPIE Conference Astronomical Telescopes and Instrumentation, Glasgow, 2004

16. Van den Dool T.et al., « The design of a breadboard cryogenic optical delay line for Darwin » SPIE Conference Astronomical Telescopes and Instrumentation, Vol. 5495-40, 2004

17. Serabyn E. and Colavita M.M., «Fully symmetric nulling beam combiners », Applied Optics, Vol.40, No 10, pp 1668-1671, 2001

18. Mitachi \& al. «Refractive index dispersion for BaF2-GdF3-ZrF4-AlF3 glasses », Applied Optics, Vol.22, No 16, pp 2419-2425, 1983

19. Maze \& al. « Fluorid glass fibers for light transmission up to $5 \mu \mathrm{m}$ », SPIE Vol. 484, p 93-98, 1984

20. Le Duigou J.M., Poupinet A., « The MOUSE II sensors for longitudinal measurements », Proceedings of OPTRO 2005, 2005

21. W.C. Danchi et al, « The Fourier-Kelvin stellar interferometer : a practical interferometer for the detection and characterization of extra-solar giant planets », SPIE Vol 5491 p236 à 241, 2004 